\title{
Aspiring to Treat Wisely: Challenges in Diagnosing and Optimizing Antibiotic Therapy for Aspiration Pneumonia
}

\author{
Christopher J Russell, MD, MS ${ }^{1,2 *}$, Andrew A White, MD³
}

'Division of Hospital Medicine, Children's Hospital Los Angeles, Los Angeles, California; ${ }^{2}$ Department of Pediatrics, Keck School of Medicine, University of Southern California, Los Angeles, California; ${ }^{3}$ Division of General Internal Medicine, Department of Medicine, University of Washington, Seattle, Washington.

$\mathrm{n}$ this issue of the Journal of Hospital Medicine, Dr. Thomson and colleagues present an analysis of 4,700 hospitalizations in the Pediatric Health Information System (PHIS) database to compare the effectiveness of different antibiotic regimens for children with neurological impairment and aspiration pneumonia. ${ }^{1}$ After adjusting for potential confounders, including illness severity markers and demographic factors, they observed that receiving anaerobic coverage was associated with improvements in rates of acute respiratory failure, intensive care unit (ICU) transfer frequency, and length of stay. Given that the authors used an administrative database, several considerations limit the generalizability of the current study. These limitations include that only patients hospitalized at freestanding children's hospitals were included, the incomplete ability to assess illness severity, and the absence of validated clinical criteria for the diagnosis of aspiration pneumonia. Despite the limitations of a retrospective study using administrative data, the authors should be commended for their rigorous analyses and for their important contribution to the care of this understudied population.

Optimizing appropriate antibiotic therapy for children with suspected aspiration pneumonia is challenging for several reasons. First, previous epidemiological studies demonstrated that viruses cause most pediatric community-acquired pneumonia'; however, we lack tools to identify patients who do not require antibiotic therapy. Second, current clinical guidelines on community-acquired pneumonia do not address aspiration pneumonia diagnosis and management. ${ }^{3}$ Similar to community-acquired pneumonia, aspiration pneumonia is a clinical diagnosis supported by patient history and laboratory and radiographic data. Given the lack of a gold standard, diagnosis of aspiration pneumonia is difficult to confirm. Previous studies using the PHIS database have demonstrated that, compared with children with nonaspiration pneumonia, those with aspiration pneumonia International Classification of Diseases, Ninth Revision, Clinical Modification (ICD-9-CM) codes feature higher rates of mortality, ICU-level care, and 30-day readmission rates. ${ }^{4,5}$ However, in

*Corresponding Author: Christopher J. Russell, MD; Email: crussell@chla.usc. edu; Telephone: 323-361-6177; Twitter: @cjrussellMD.

Received: December 5, 2019; Revised: December 12, 2019;

Accepted: December 26, 2019

๑) 2020 Society of Hospital Medicine DOI 10.12788/jhm.3375 these studies, patients with an ICD-9-CM code for aspiration pneumonia were also more medically complex, with a higher number of complex chronic conditions and rates of technology use. Lastly, aspiration pneumonia is occasionally synonymous with pneumonia in medically complex patients, which leads to the increased exposure to broad-spectrum antibiotics. The exposure to broad-spectrum antibiotics causes complications, such as Clostridioides difficile infection and potential antibiotic resistance in a patient population that already experiences significant antibiotic exposure.

Growing concerns about antibiotic overuse and the declining prevalence of anaerobic isolates among adult pneumonia patients recently prompted the Infectious Diseases Society of America (IDSA) and the American Thoracic Society (ATS) to discourage routine anaerobic coverage among adults with suspected aspiration pneumonia and no abscess or empyema. ${ }^{6}$ These guidelines overturn years of habit for most adult hospitalists, although the IDSA and ATS acknowledge the extremely low quality of evidence informing the recommendation. Thus, the dilemma is whether the IDSA/ATS guidelines should be reconciled with the conclusions of Thomson et al. The answer is "not necessarily." Fundamentally, different causes of neurological impairment, such as dementia and stroke, afflict elderly adults with aspiration pneumonia along with important differences in physiological and microbiological exposures. Instead, adult and pediatric hospitalists can find common ground around the shared uncertainty and variability in diagnosing aspiration pneumonia and the need for more credible evidence. Unfortunately, wide variation in diagnosis and coding practices might complicate the efforts to reproduce Thomson's rigorous retrospective cohort study in large adult databases ${ }^{7}$ given that Medicare-quality comparison programs may have inadvertently encouraged changes in coding behaviors during the last decade. Attributing pneumonia cases to aspiration removed high-risk patients from reporting cohorts, thus improving a hospital's apparent mortality rate for community-acquired pneumonia. Although the United States Centers for Medicare \& Medicaid Services amended rules in 2017 to address this concern, years of overdiagnosis of aspiration pneumonia possibly biased adult administrative data sets.

Although the association between the use of anaerobic antibiotic coverage and improved pediatric outcomes is promising, these results also point out the need for rigorous prospective studies to improve the evidence base for the di- 
agnosis and treatment of suspected aspiration pneumonia in hospitalized patients of all ages. Given the heterogeneity in the use of aspiration pneumonia diagnoses, foundational work might include assessing the factors that influence clinicians in deciding on the diagnosis of aspiration pneumonia (versus community-acquired pneumonia). On the patient side, parallel trials may start with multicenter, prospective cohort studies to gain insights into the demographic, clinical, and laboratory factors that are associated with the diagnosis of aspiration pneumonia. This research direction may lead to the development and standardization of diagnostic criteria for aspiration pneumonia. Ultimately, prospective randomized controlled trials are needed to assess the comparative effectiveness of different antibiotic choices on clinical outcomes.

Disclosures: The authors have nothing to disclose.

\section{References}

1. Thomson J, Hall M, Ambroggio L, et al. Antibiotics for aspiration pneumonia in neurologically impaired children. J Hosp Med. 2020;15(7):395-402. https:// doi.org/10.12788/jhm.3338

2. Jain $S$, Williams DJ, Arnold $S R$ et al. Community-acquired pneumonia requiring hospitalization among U.S. children. N Engl J Med. 2015;372(9):835845. https://doi.org/10.1056/NEJMoa1405870

3. Bradley JS, Byington $\mathrm{CL}$, Shah SS, et al. The management of community-acquired pneumonia in infants and children older than 3 months of age: clinical practice guidelines by the Pediatric Infectious Diseases Society and the Infectious Diseases Society of America. Clin Infect Dis. 2011;53(7):e25-76. https://doi.org/10.1093/cid/cir531

4. Hirsch AW, Monuteaux MC, Fruchtman G, Bachur RG, Neuman MI. Characteristics of children hospitalized with aspiration pneumonia. Hosp Pediatr. 2016;6(11):659-666. https://doi.org/10.1542/hpeds.2016-0064

5. Thomson J, Hall M, Ambroggio $L$, et al. Aspiration and non-aspiration pneumonia in hospitalized children with neurologic impairment. Pediatrics. 2016;137(2):1-10. https://doi.org/10.1542/peds.2015-1612

6. Metlay JP, Waterer GW, Long AC, et al. Diagnosis and treatment of adults with community-acquired pneumonia. An official clinical practice guideline of the American Thoracic Society and Infectious Diseases Society of America. Am J Respir Crit Care Med. 2019;200(7):e45-e67. https://doi.org/10.1164/ rccm.201908-1581ST

7. Lindenauer PK, Strait KM, Grady JN, et al. Variation in the diagnosis of aspiration pneumonia and association with hospital pneumonia outcomes. Ann Am Thorac Soc. 2018;15(5):562-569. https://doi.org/10.1513/AnnalsATS.201709-728OC 